\title{
FlowPGA: DataFlow de Aplicações em FPGA
}

\author{
Leandro A. J. Marzulo, Fabio H. Flesch, Alexandre S. Nery, Felipe M. G. França, Edil S. T. Fernandes \\ Universidade Federal do Rio de Janeiro \\ Programa de Engenharia de Sistemas e Computação, COPPE \\ Rio de Janeiro - Brazil \\ $\{$ lmarzulo, fabioflesch, solon, felipe, edil $\} @$ cos.ufrj.br
}

\section{Resumo}

A arquitetura WaveScalar é a primeira arquitetura dataflow a apresentar uma interface de memória que mantém a semântica de acessos requerida pelas linguagens imperativas. Um protótipo da arquitetura, em desenvolvimento, permitiria passar de experimentação por simulação para um cenário mais real, com o processador desenvolvido em FPGA. No entanto, este protótipo não é acessível (financeiramente) para qualquer instituição que também queira produzí-lo. Neste trabalho é apresentada a FlowPGA, uma versão reduzida desta arquitetura para ser utilizada com FPGAs com pequeno número de células lógicas. Uma FPGA com 1,5 milhões de gates foi utilizada para implementação. A corretude da implementação foi avaliada com a execução de um programa de multiplicação entre dois números positivos usando sucessivas somas. Os resultados mostram que a arquitetura FlowPGA tem desempenho equivalente ao WaveScalar. Ainda, para avaliar a versatilidade do projeto, a FlowPGA foi modificada para utilizar um sistema de numeração RNS, com esforço de implementação de aproximadamente 20 horas.

\section{Introdução}

Com a popularização de máquinas multi-processadas (e multi-cores), extrair paralelismo de aplicações vem se tornando uma preocupação cada vez mais importante para atingir alto desempenho. O uso do modelo de Von Newmann, com sua natureza sequencial e baseada no fluxo de controle, dificulta esta tarefa, motivando importantes pesquisas sobre modelos alternativos, como TRIPS [5, 8] e Raw [14]. Outra alternativa a esse cenário é o modelo Dataflow, onde as instruções são executadas assim que tenham disponíveis os operandos de entrada necessários, ao invés de seguir a ordem do programa. Como é possível ter diversas instruções no pipeline provenientes de diversos contextos, os problemas causados por dependências de dados e/ou de controle podem ser evitados [9]. Infelizmente as máquinas realmente Dataflow, nunca foram populares, principalmente porque elas não suportavam a semântica de memória requerida pelas linguagens imperativas, exigindo uma nova arquitetura e linguagem de programação para migrar para este modelo.

A arquitetura WaveScalar proposta por Swanson é uma releitura dos conceitos Dataflow, para endereçar este problema, provendo uma interface de memória que realiza os acessos respeitando a ordem do programa [10, 11, 12]. A idéia chave é que a computação é dividida em ondas (waves), tal que cada onda tem a execução guiada pelo fluxo de dados, mas o sequenciamento de ondas garante a tradicional ordem de acesso à memória. Com isto, foi possível executar programas imperativos em uma máquina Dataflow, e ainda obter speedups significativos. Para aplicações single-threaded o WaveScalar apresenta desempenho equivalente a um superescalar ou a um CMP, com economia de $30 \%$ em área de silício. Para aplicações multi-threaded o WaveScalar apresenta speedups de 2 a 11, em relação a $C M P s[10]$.

O projeto $R A M P$ [1] propõe a criação de uma plataforma de emulação baseada em FPGA (Field-Programable Gate Array) para acelerar a pesquisa em multiprocessadores. A intenção é que os grupos de pesquisa em computação paralela adotem a prototipagem para avaliar suas idéias ao invés de usarem apenas simuladores. O projeto WaveScalar é citado no RAMP como um dos possíveis grupos a sererm beneficiados pelo uso desta plataforma.

No protótipo do WaveScalar [6], em desenvolvimento, estão sendo usadas 16 placas de circuito, cada qual com 4 FPGAs Virtex II Pro (Xilinx®). Em cada FPGA é representado um Domain, com oito Processing Elements, além de porções do switch e StoreBuffer. É visível que este tipo de projeto não está acessível a qualquer centro de pesquisa. A principal motivação deste trabalho é criação de uma uma versão simplificada da arquitetura WaveScalar, que possa ser mapeada em FPGAs com baixo número de 
células reconfiguráveis. $\mathrm{O}$ uso da linguagem Handel-C [7] permite descrever o projeto em um nível mais alto, possiblitando que adaptações para adequá-lo a diferentes necessidas sejam realizadas, de forma simples e rápida.

A Seção 2 descreve o conjunto de instruções e a arquitetura do WaveScalar. A Seção 3 apresenta a arquitetura FlowPGA e discute alguns detalhes de implementação, bem como as ferramentas desenvolvidas para facilitar a criação de programas para a FlowPGA. A Seção 4 apresenta os experimentos realizados para verificar a corretude do projeto, avaliar a funcionalidade do conjunto de ferramentas e também a facilidade de alterar o projeto para adaptá-lo a outras necessidades (neste caso o uso do sistema RNS (Residue Number System) de numeração, aplicado frequentemente em processamento de sinais). A Seção 5 apresenta as conclusões e trabalhos futuros.

\section{WaveScalar}

O WaveScalar $[10,11,12]$ foi a arquitetura dataflow usada como base na implementação da FlowPGA, apresentada neste trabalho. Esta Seção provê uma breve descrição do conjunto de instruções e arquitetura deste processador.

\subsection{O Conjunto de Instruções}

Um grafo de fluxo de dados é utilizado para descrever um programa no modelo DataFlow. Os nós no grafo são as instruções, que são inteligentes, no sentido de que estão associadas a uma unidade funcional. As arestas representam operandos trocados entre instruções. O conjunto de instruções do WaveScalar é derivado do conjunto de instruções da máquina Alpha [3]. A principal diferença é que os desvios devem ser transformados em um mecanismo que seleciona os consumidores dos valores (operandos): (i) a instrução Select $(\phi)$ recebe dois valores $v_{1}$ e $v_{2}$ e um booleano $s$, que seleciona um dos valores; a instrução (ii) Steer $(\rho)$ recebe um valor $v$ e um booleano $b$ que define um de dois possíveis destinos para o envio de $v$.

\subsubsection{Ondas}

As ondas (waves) são fragmentos acíclicos e conexos do grafo de fluxo de controle com uma única entrada, estendendo os hiper-blocos, pois também podem possuir junções. As diferentes iterações em um laço podem executar em paralelo no modelo dataflow, caso não haja dependências entre suas instruções. Dada uma instrução em um laço e um operando enviado para a mesma, a instância da instrução de destino é indicada por um rótulo no operando, que marca o número da onda (ou iteração). A instrução Wave-Advance (WA) incrementa este número para cada operando de entrada de uma onda. A Figura 1 mostra o grafo dataflow (a) associado a um trecho de programa (b). As ondas estão envolvidas por linhas pontilhadas.

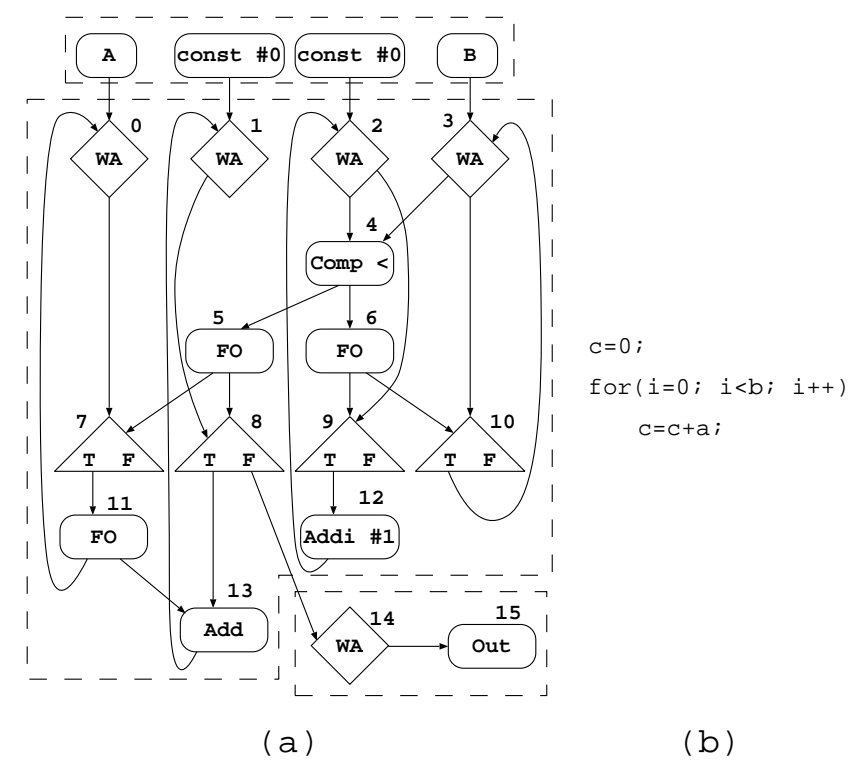

Figura 1. Grafo dataflow do WaveScalar.

\subsubsection{Wave-ordering annotations}

Durante o processo de compilação todas as operações de memória recebem uma chave $\langle P, C, S\rangle$ (Predecessor, Corrente e Sucessor), permitindo que o subsistema de memória estabeleça uma cadeia conectando-as em uma onda. Toda requisição de acesso à memória é enviada para a interface de memória, inserida em um buffer e só poderá ser atendida se todas as requisições anteriores na cadeia e todas as ondas anteriores já tiverem sido executadas.

\subsection{A Arquitetura WaveScalar}

A arquitetura WaveScalar é composta por um conjunto de elementos de processamento (PEs) idênticos, o hardware da Wave-ordered memory e uma rede hierárquica para suportar a comunicação. O bloco de construção da WaveCache, é o Cluster, que possui uma cache L1, o StoreBuffer que faz a interface com a Wave-ordered memory, e um Switch que prove comunicação inter e intra-Cluster. Cada Cluster tem quatro Domains, que por sua vez possuem oito elementos de processamento agrupados em Pods de dois PEs cada. Os Clusters são replicados no die, formando uma matriz que é conectada à cache L2 em suas bordas. A Figura 2 (reproduzida com 
permissão do autor, de [10]) mostra uma visão geral da WaveCache.

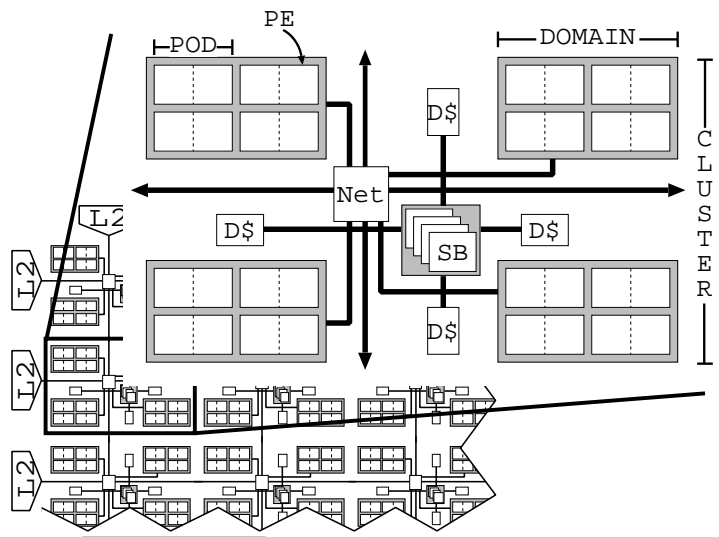

Figura 2. A arquitetura WaveCache

Os PEs implementam a regra de disparo Dataflow e a execução de instruções. Cada $P E$ tem a sua $A L U$, estruturas de memória para armazenar operandos, lógica de controle de execução e comunicação e um buffer de instruções. Quando um programa é executado no WaveScalar, múltiplas instruções são mapeadas em um mesmo $P E$, segundo um algoritmo de placement. Conforme a evolução da execução do programa, algumas instruções tornam-se desnecessárias e são substituídas por outras. A regra de disparo garante que uma instrução é executada quando todos os seus operandos de entrada estão disponíveis. Cada $P E$ possui também uma Matching Table que armazena operandos destinados à instruções mapeadas naquele $P E$, até que as mesmas estejam prontas para serem disparadas. Quando isto ocorre, tais operandos são consumidos, produzindo resultados que serão enviados para outras instruções (em PEs remotos ou no $P E$ local). $\mathrm{O} P E$ possui um pipeline de cinco estágios, com redes de bypass que permitem a execução de instruções dependentes no mesmo $P E$. A Figura 3 mostra a arquitetura de um $P E$, destacando os estágios de seu pipeline, descritos a seguir:

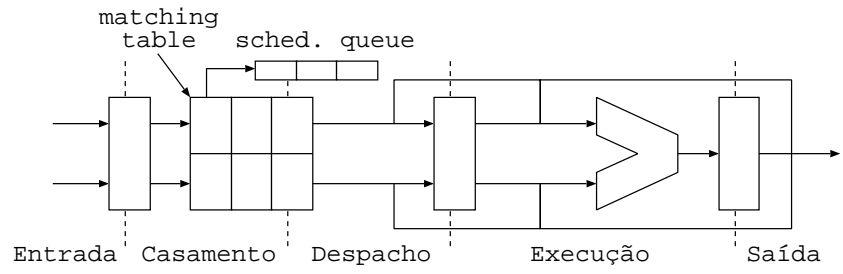

Figura 3. Um Processing Element

Entrada: Chegada de operandos no $P E$, enviados por outro $P E$ ou pelo próprio através da rede de bypass.

Casamento de tag dos operandos (Match): Os operandos são colocados em uma tabela chamada matching table, onde suas tags são verificadas em busca de um casamento de operandos para uma mesma instrução e onda. Quando uma instrução já possui todos os seus operandos disponíveis é movida para uma fila de instruções prontas para execução, chamada scheduling queue. O casamento também pode ocorrer especulativamente, quando o $P E$ supõe que alguma instrução que está executando localmente produzirá operandos para uma outra, também local.

Despacho: O PE seleciona uma instrução da scheduling queue, lê seus operandos da matching table e os envia para o estágio de execução.

Execução: Executa a instrução e envia os resultados para o estágio de saída, exceto quando: (i) a mesma foi disparada especulativamente e ainda não possui todos os operandos de que necessita; (ii) o buffer de saída está cheio. No primeiro caso, a instrução é eliminada da scheduling queue e no segundo ocorre um stall no estágio de execução até que haja espaço disponível.

Saída: Os resultados da instrução são enviados pelo barramento de saída para o próprio $P E$ ou outro remoto. É feita a difusão da informação pelo barramento que conecta os PEs em um Domain, usando um protocolo de transmissão ACK/NACK.

\section{A FlowPGA}

Nesta seção é descrita a arquitetura FlowPGA e seu conjunto de instruções, os detalhes da implementação em FPGA, a linguagem de descrição de grafos dataflow, bem como o conjunto de ferramentas (tradutor e visualizador de mensagens).

\subsection{Conjunto de Instruções e Arquitetura}

A arquitetura FlowPGA é baseada, com algumas simplificações, na arquitetura WaveScalar, permitindo o mapeamento em FPGAs com baixo número de células reconfiguráveis e viabilizando o estudo de arquiteturas dataflow, em um ambiente real de execução. Nesta versão ainda não está contemplada a interface de acesso à memória (Wave-ordered memory) e um mecanismo de placement dinâmico que permita carregar programas com mais instruções estáticas do que o suportado pelas listas de instruções dos PEs. Esse mecanismo faria o swap de instruções da lista, conforme a evolução do programa em execução. O swapping de operandos da Matching Table e 


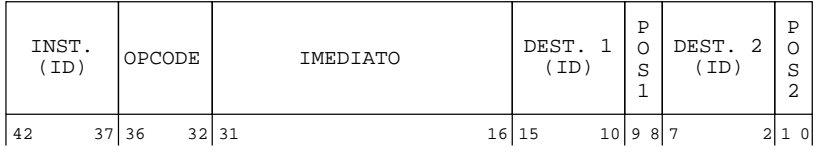

Figura 4. Formato das instruções

\section{Tabela 1. Significado dos campos de posição}

\begin{tabular}{c|l}
\hline 0 & Campo destino correspondente não é utilizado \\
\hline 1 & Operando direito \\
\hline 2 & Operando esquerdo \\
\hline 3 & Único operando \\
\hline
\end{tabular}

listas de espera também não é contemplado nesta versão. Sendo assim, o sistema não está preparado para execução de programas com alto grau de paralelismo, que esgotariam o espaço de armazenamento de operandos.

As instruções da FlowPGA possuem um tamanho fixo e regular, para facilitar a decodificação. Cada instrução contém um número identificador (ID), o opcode que indica a operação a ser realizada na ALU, um imediato, além dos IDs das instruções de destino. Nesta versão do FlowPGA cada instrução pode receber 1 ou 2 operandos e enviar resultados para até 2 instruções. Caso seja necessário enviar o resultado para mais de dois destinos, é usada a instrução FANOUT que recebe um operando de entrada e encaminha para dois destinos.

Dependendo da instrução, a posição dos operandos de entrada é relevante para a produção do resultado (em uma divisão, por exemplo, é necessário saber quem são o divisor e o dividendo). Sendo assim, para cada um dos 2 destinos de uma instrução é informada também a posição. A Figura 4 exibe o formato de uma instrução da FlowPGA, com todos os seus campos, e as Tabelas 1 e 2 relacionam os valores para os campos posição e opcode, com seus respectivos significados.

Tabela 2. Significado do campo opcode

\begin{tabular}{r|l}
\hline 0 & Soma \\
\hline 1 & Subtração \\
\hline 2 & Multiplicação \\
\hline 3 & Divisão \\
\hline 4 & Mod \\
\hline 5 & E lógico \\
\hline 6 & Ou lógico \\
\hline 7 & Negação lógica \\
\hline 8 & Comparação $>$ \\
\hline 9 & Comparação $<$ \\
\hline 10 & Comparação $=$ \\
\hline 11 & Steer \\
\hline 12 & Wave-Avance \\
\hline
\end{tabular}

\begin{tabular}{l|l}
\hline 13 & FanOut \\
\hline 14 & Saída de Resultado (USB) \\
\hline 16 & Soma com imediato \\
\hline 17 & Subtração com imediato \\
\hline 18 & Multiplicação com imediato \\
\hline 19 & Divisão com imediato \\
\hline 20 & Resto com imediato \\
\hline 21 & E lógico com imediato \\
\hline 22 & Ou lógico com imediato \\
\hline 24 & Comparação > Imediato \\
\hline 25 & Comparação < Imediato \\
\hline 26 & Comparação = Imediato \\
\hline & \\
\hline
\end{tabular}

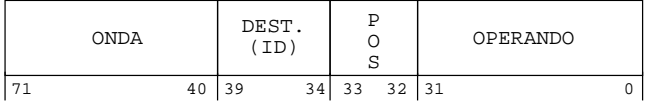

Figura 5. Mensagens entre instruções

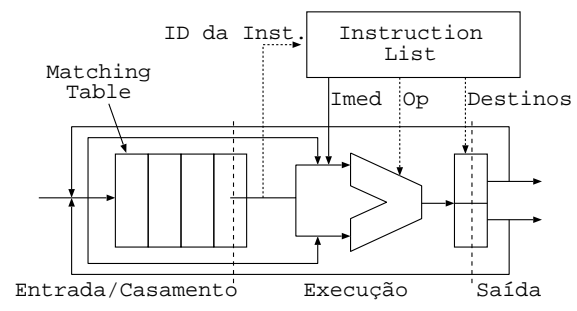

Figura 6. Um PE na FlowPGA

A execução de uma instrução gera operandos de saída que podem ser enviados para outras instruções, mapeadas em qualquer $P E$. Os operandos são enviados em forma de mensagens contendo o número da onda, ID da instrução de destino, posição do operando na instrução destino e valor do operando. O formato da mensagem é exibido na Figura 5.

Cada Processing Element da FlowPGA foi implementado como um pipeline assíncrono de 3 estágios. A Figura 6 exibe o $P E$, cujos estágios são descritos a seguir:

Entrada e Casamento: Chegada de no máximo 1 operando por ciclo no $P E$, enviados por outro $P E$ ou pelo próprio através da rede de bypass. A mensagem de entrada é: (i) enviada para execução, se o campo Posição da mensagem possui o valor 3; (ii) enviada para execução juntamente com outro operando da Matching Table, se ocorrer um casamento; (iii) enviada para uma fila de espera, caso a Matching Table esteja cheia; (iv) inserida na Matching Table, caso o campo posição seja 1 ou 2, e não tenha ocorrido casamento.

Execução: Acessa a instrução indicada na Instruction List, realiza a execução e envia os resultados para o estágio de saída.

Saída: Os resultados da instrução são inseridos em um buffer de saída e enviados pelo barramento de saída para o próprio $P E$ (pela rede de bypass) ou outro remoto (por um switch Butterfly [2]). Uma mensagem é produzida para cada destino. O PE pode seguir com a execução de instruções, pois cada mensagem só será apagada do buffer quando tiver sido recebida pelo switch, que garantirá a sua entrega.

Percebe-se que não há necessidade da scheduling queue, pois não há execução especulativa e, além disso, como só 


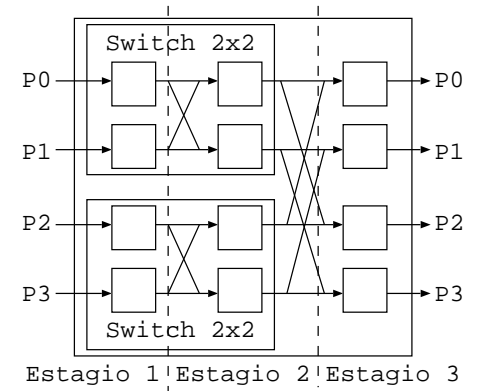

Figura 7. Switch Butterfly $4 \times 4$

uma mensagem é recebida por ciclo, todo o casamento gera uma execução imediata. Pelo mesmo motivo, só é necessário armazenar, no máximo, um operando por instrução na Matching Table, ficando a mesma reduzida a linhas contendo apenas o número da onda, número da instrução, operando e posição. Como a Matching Table é percorrida em paralelo no estágio de casamento, ela deve ter um número pequeno de linhas para que esta tarefa não seja cara. Daí a existência da lista de espera. Também não é necessário o estágio de Despacho, pois não há scheduling qиеие е a rede de bypass envia operandos apenas para o estágio de Entrada/Casamento.

A comunicação entre os PEs é feita por intermédio de um Switch Butterfly, descrito na Figura 7. Este é implementado com um pipeline sincrono de 3 estágios, sendo cada um responsável pela execução de um hop até a entrega da mensagem ao Destino. É importanete salientar que no WaveScalar, a comunicação entre PEs de um mesmo Domain é feita ponto-a-ponto. Embora isto possibilite um maior paralelismo, na presente arquitetura, optou-se pela alternativa de alterar o switch para simplificar o projeto e facilitar a escalabilidade do mesmo (inserir novos PEs). O Switch Butterfly 4x4 é construído recursivamente a partir de switches $2 \times 2$. Para ampliá-lo, basta seguir o mesmo princípio. Para saber qual processador deve receber uma determinada mensagem, o switch consulta uma tabela de roteamento que guarda uma lista dos PEs, associados a todas as instruções mapeadas.

\subsection{Implementação em FPGA}

A arquitetura FlowPGA, descrita na Seção 3, foi implementada utilizando a linguagem Handel-C, tendo como alvo a placa $\mathrm{RC10}$, ambas desenvolvidas

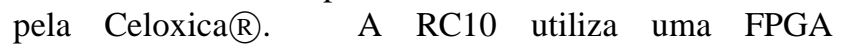
Xilinx@Spartan-3E, que pode operar com um clock entre 2 e $300 \mathrm{MHz}$. Ela possui 1,5 milhões de células lógicas e disponibiliza uma interface de comunicação via USB, além de uma série de dispositivos de E/S (VGA, teclado, mouse, display de sete segmentos, leds, entre outros). A implementação desta arquitetura se deu por meio do kit de desenvolvimento DK5, também licenciado

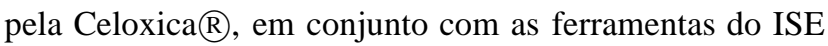
WebPack da Xilinx $囚$.

A comunicação entre PEs e Switch é feita através de canais com FIFOs para armazenar até sete mensagens em cada. As estruturas FIFO atuam como os reject buffers do WaveScalar. Para a comunicação entre os estágio do pipeline assíncrono de cada $P E$ foram utilizados canais sem FIFO. Uma interface de recebimento de mensagens externas através da USB foi elaborada para fazer a carga de operandos de inicialização da computação. A instrução OUT, descrita na Seção 3.1 gera uma mensagem de saída contendo o operando, que é encaminhada para a interface USB e pode ser coletada pelo PC hospedeiro. A Figura 8 mostra uma visão geral da arquitetura FlowPGA (envolvida pela linha pontilhada) e sua comunicação com o PC.

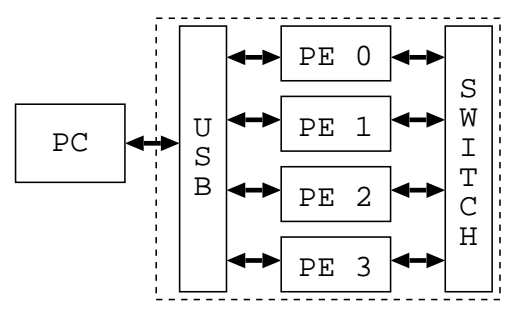

Figura 8. Visão geral da FlowPGA

A implementação de $A L U s$ com capacidade para executar todos os tipos de instrução descritos na Tabela 2 só seria possível, para a placa RC10, em alternativas de design menos eficientes. Neste trabalho, houve uma preocupação maior em criar uma versão da FlowPGA com desempenho mais próximo possível da arquitetura WaveScalar. Desta forma, o projeto foi otimizado ao máximo, dentro dos limites da placa. As operações de divisão e multiplicação são as que ocupam a maior área no projeto e também as que demandam o ciclo mais longo para execução. Estas operações não foram incluídas nesta implementação, resultando em um projeto que ocupa aproximadamente quinhentos e cinqüenta mil células lógicas e pode executar em freqüências de até $48 \mathrm{MHz}$. Vale lembrar que esta é uma das possíveis alternativas de design. A descrição da arquitetura em uma linguagem de alto nível, como o Handel-C, facilita a adequação do projeto para outras necessidades. Na Seção 4.2 é feita a descrição de uma versão do FlowPGA para usar o sistema RNS [4, 13].

\subsection{Descrição do grafo dataflow}

Para facilitar a escrita de programas para a FlowPGA, foi desenvolvida uma linguagem para descrever o grafo 
dataflow e definir o placement das instruções nos PEs. Nesta, informa-se a quantidade de $P E s$, as instruções a serem executadas por cada $P E$ e os dados de entrada do programa. A Figura 9 mostra o programa FlowPGA associado ao grafo dataflow da Figura 1.

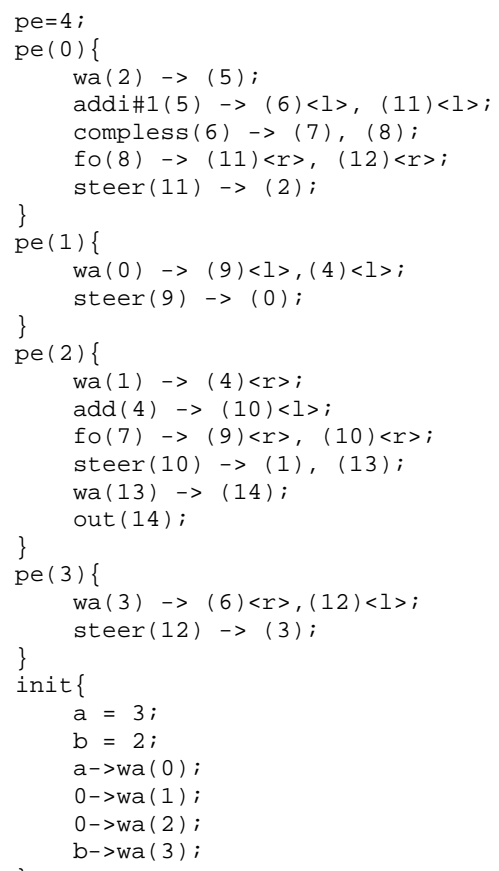

Figura 9. Um programa FlowPGA

Primeiramente, a tabela de roteamento associa cada instrução estática a um $P E$. Um vetor armazena uma lista de números de $P E$ s indexada pelo identificador da instrução. Dentro de cada bloco pe(id), uma instrução corresponde a uma linha que obedece ao formato:

$$
\text { opcode\#im }(i d) \rightarrow(i d)<\text { side }>,(i d)<\text { side }>\text {; }
$$

Neste formato, o resultado da operação indicada à esquerda da seta será enviado para as instruções de destino indicadas à direita da mesma. Além disso, este resultado poderá ser inserido no operando direito ou esquerdo destas instruções, conforme indicado pelo campo $<$ side $>$. Caso nada seja informado a respeito da posição de inserção, o tradutor assumirá que a instrução de destino admite apenas um operando. Algumas instruções também permitem operandos imediatos, indicados no campo \#im.

$\mathrm{O}$ último bloco, denominado init, inicializa a computação enviando mensagens para cada um dos PEs descritos nos blocos anteriores. As mensagens podem conter constantes de inicialização ou variáveis, cujos valores devem ser atribuídos antes do envio.

\subsection{Ferramentas}

Uma ferramenta de tradução foi criada para a especificação de um programa FlowPGA. Ela tem como entrada um grafo dataflow, descrito de acordo com a linguagem especificada na Seção 3.3, e como saída o código binário correspondente. Um visualizador de mensagens também foi criado para separar em campos e exibir, de forma inteligível, as mensagens enviadas pela FlowPGA e coletadas pelo PC (via USB).

\section{Experimentos e resultados}

A Seção 4.1 provê uma avaliação da arquitetura FlowPGA e sua semelhança comportamental em relação ao WaveScalar. Para avaliar a versatilidade da FlowPGA, a solução original foi modificada, para que a arquitetura possa adotar um sistema de numeração $R N S$, resultando na FlowPGA-RNS. A Seção 4.2 descreve este sistema de numeração e a Seção 4.3 detalha os experimentos realizados para esta alternativa de design.

\subsection{Avaliação da FlowPGA}

A linguagem Handel-C permite implementar rapidamente uma solução em FPGA, mas como a descrição é feita em alto nível, o produto final pode não ser o mais otimizado. Uma solução em Handel-C é mais genérica que uma solução em uma linguagem de descrição de hardware (como VHDL ou Verilog). Além disso, no Handel-C, cada atribuição a uma variável leva um ciclo de clock. Sendo assim, cada estágio do pipeline de um $P E$ pode levar mais de um ciclo, embora o trabalho realizado em cada ciclo seja inferior ao de uma implementação descrita em HDL.

Com o objetivo de avaliar o grau de equivalência entre as duas implementações e validar a solução construída, o algoritmo da Figura 1 (que realiza uma multiplicação usando sucessivas somas) foi descrito como um grafo dataflow (de acordo com o modelo exposto na Seção 3). O tradutor, descrito na Seção 3.4, foi usado na elaboração do binário para a arquitetura FlowPGA. O programa foi mapeado manualmente nas listas de instruções dos processadores e, em seguida, o projeto foi compilado e gravado na FPGA. O algoritmo foi executado na FlowPGA e no simulador WaveScalar, variando o multiplicador (valor de $B$ ) entre cinqüenta e cem mil. O simulador foi configurado com as mesmas características da implementação da FlowPGA (1 Cluster com 1 Domain de 4 PEs, com até 16 instruções cada).

A Figura 10 mostra os resultados deste experimento, observando-se que as implementações são equivalentes. Cada estágio do pipe assíncrono da FlowPGA leva entre 2 e 4 ciclos. Sendo assim, uma distância quase constante, 
de aproximadamente quatro vezes, é verificada no número de ciclos da execução entre as arquiteturas. É importante lembrar que, em uma solução descrita em HDL, cada estágio pode ser projetado para durar apenas um ciclo.

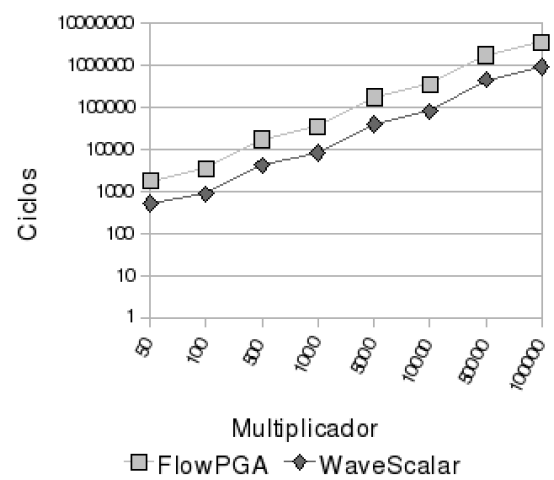

Figura 10. FlowPGA vs. WaveScalar

\subsection{RNS}

O RNS (Residue Number System) é um sistema numérico de inteiros cujas operações de adição, subtração e multiplicação são decompostas em sub-operações equivalentes que podem ser executadas em paralelo. Conseqüentemente, o RNS é conveniente para aplicações que utilizam tais operações com alta freqüência, como em processamento digital de sinais. Por outro lado, as demais operações são mais complexas e lentas.

Um sistema baseado em resíduos é construído a partir de um conjunto de $N$ números primos relativos entre si, chamados de módulos. Ou seja, para todos os pares de módulos $\left(P_{i}, P_{j}\right)$, tal que $i \neq j$, tem-se que o máximo divisor entre eles é 1.

Seja um conjunto $P=\left\{P_{0}, P_{1}, \ldots, P_{n}\right\}$ uma base RNS, conforme a definição acima. A faixa dinâmica $M$, isto é, o maior número inteiro que pode ser representado em RNS, é determinada pelo produto dos $N$ módulos que compõem a base. Logo, para o conjunto P, tem-se:

$$
M=P_{0} \cdot P_{1} \cdot \ldots \cdot P_{n}
$$

Então, dois números decimais $\mathrm{A}$ e $\mathrm{B}$ positivos terão como representação em RNS as $N$-tuplas $A_{R N S}=$ $\left(A_{0}, A_{1}, \ldots, A_{n}\right)$ e $B_{R N S}=\left(B_{0}, B_{1}, \ldots, B_{n}\right)$, dadas por $A_{i}=|A|_{P_{i}}$ e $B_{i}=|B|_{P_{i}}$. A notação $|A|_{P_{i}}$ equivale a $A$ mod $P_{i}$. Para números negativos é necessário fazer uma operação de complemento (mais detalhes podem ser obtidos em $[4,13])$.

Operações de adição, subtração e multiplicação entre $A_{R N S}$ e $B_{R N S}$, têm como resultado uma $N$-tupla $R=$
$\left(R_{0}, R_{1}, \ldots, R_{n}\right)$, dada por $R_{i}=\mid A_{i}$ op $\left.B_{i}\right|_{P_{i}}$, lembrando que o resultado $A_{R N S}$ op $B_{R N S}=R$ deve estar dentro da faixa dinâmica estabelecida por $M$.

Uma vez que os pares de módulos $\left(P_{i}, P_{j}\right)$ são primos relativos entre si, pode-se usar o Teorema Chinês do Resto $[4,13]$ a fim de converter um número do sistema de resíduos para o sistema numérico convencional. Para a base $P$, cuja faixa dinâmica corresponde a $M$, deseja-se obter o valor decimal de $R$. Pelo Teorema Chinês do Resto:

$$
R=\left|\sum_{i=0}^{3}\left(\left|P_{i}^{\prime} \cdot R_{i}\right|_{P_{i}} \cdot\left(\frac{M}{P_{i}}\right)\right)\right|_{M}
$$

Os coeficientes $P_{i}^{\prime}$ são constantes que podem ser computadas uma única vez e armazenadas. Tais constantes são calculadas encontrando-se o valor de $x_{i}$ para o qual:

$$
\left|\left(\frac{M}{P_{i}}\right) \cdot x_{i}\right|_{P_{i}}=1
$$

\subsection{Avaliação da FlowPGA-RNS}

A corretude da FlowPGA-RNS foi avaliada também com o programa da Figura 1. Os multiplicadores foram variados entre dois e mil, e a execução foi realizada para ambas arquiteturas (FlowPGA e FlowPGA-RNS). O conjunto de co-primos utilizado foi $P=\{32,29,27,23\}$, resultando em uma faixa dinâmica $M=576288$. Como o maior valor para um componente RNS deste sistema é 31, a solução foi preparada para trabalhar com operandos de 5 bits. As $A L U s$ também foram alteradas para realizar apenas operações de soma, subtração e multiplicação (com e sem imediato), no formato RNS. Foram necessárias aproximadamente vinte horas para modificar a solução original e gerar a FlowPGA-RNS, o que confirma a versatilidade da arquitetura e da linguagem Handel-C.

A Figura 11 mostra a comparação de desempenho entre estas alternativas de design. Observa-se que para multiplicadores pequenos a FlowPGA sobrepuja a FlowPGA-RNS. A medida que os multiplicadores aumentam, os números de ciclos de execução se aproximam e se cruzam para valores de $B$ (multiplicador, no algoritmo) entre quinze e cinqüenta. Como qualquer número em $R N S$ tem valor máximo igual a trinta e um, para suas componentes, este será o maior número de iterações executadas, no cálculo da multiplicação (por sucessivas somas). Desta forma, o número de ciclos para o FlowPGA-RNS se mantém na mesma faixa, enquanto que para a FlowPGA ocorre um aumento proporcional ao tamanho de $B$. Obviamente não esta sendo considerado o tempo de conversão decimal-RNS, pois esta tarefa é realizada antes de enviar os operandos de entrada para a FPGA. 


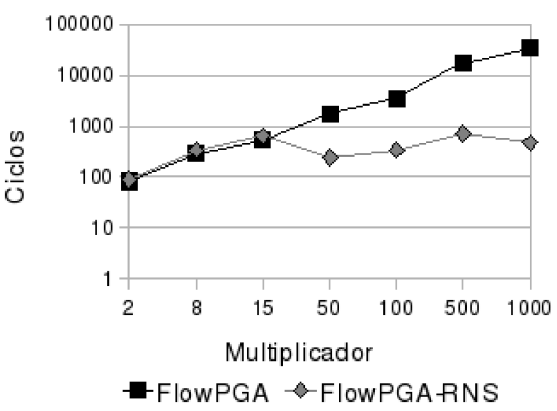

Figura 11. FlowPGA vs. FlowPGA-RNS

Um outro dado a ser observado é a redução de $9 \%$ dos recursos utilizados na $F P G A$, diante da adoção do RNS. Isto ocorre pois a FlowPGA-RNS utiliza um menor número de bits para seus operandos, simplificando também as unidades funcionais. Foi possível atingir tal redução, mesmo com a modificação destas unidades para realizar uma operação de resto da divisão, associada a cada operação aritmética já existente.

\section{Conclusões e Trabalhos Futuros}

Neste Trabalho foi apresentada a FlowPGA, uma arquitetura dataflow em FPGA, para o estudo de aplicações e outras alternativas de design para este modelo. Em experimentos realizados, foi verificada a proximidade com a arquitetura WaveScalar (usada como base). Tais experimentos mostram que a FlowPGA traduz corretamente o comportamento do WaveScalar.

A implementação em Handel-C facilita a alteração do projeto para outros propósitos. Como prova de conceito, foi contruída uma segunda versão da arquitetura, que utiliza RNS como sistema de numeração. A implementação da FlowPGA-RNS levou aproximadamente vinte horas, comprovando a versatilidade da implementação original.

Alguns trabalhos futuros decorrem deste trabalho, entre eles: (i) a criação de um conjunto de aplicações para avaliar a implementação da versão com RNS, mais detalhadamente; (ii) a inclusão de um mecanismo que permita mapear aplicações dinâmicamente, sem a necessidade de recompilar o projeto; (iii) a inclusão de uma interface de acesso à memória; e (iv) a experimentação de uma implementação em uma HDL, permitindo o uso da FlowPGA com outras FPGAs.

\section{Referências}

[1] Arvind, K. Asanovic, D. Chiou, J. C. Hoe, C. Kozyrakis, S.-L. Lu, M. Oskin, D. Patterson, J. Rabaey, and
J. Wawrzynek. Ramp: Research accelerator for multiple processors - a community vision for a shared experimental parallel hw/sw platform. Technical Report UCB/CSD-05-1412, EECS Department, University of California, Berkeley, Sep 2005.

[2] B. A. Computers. Quarterly technical report no. 3 april 1 \& july 15.1984 development of a butterfly multiprocessor test bed: The butterfly switch. Technical report, BBN Advanced Computers, Cambridge, Massachusetts, 1985.

[3] R. Desikan, D. C. Burger, S. W. Keckler, and T. Austin. Sim-alpha: A validated, execution-driven alpha 21264 simulator. Technical Report TR-01-23, UT-Austin Computer Sciences, 2001.

[4] I. Koren. Computer arithmetic algorithms. Prentice-Hall, Inc., Upper Saddle River, NJ, USA, 1993.

[5] R. Nagarajan, K. Sankaralingam, D. Burger, and S. Keckler. A design space evaluation of grid processor architectures. In Microarchitecture, 2001. MICRO-34. Proceedings. 34th ACM/IEEE International Symposium on, pages 40-51, 1-5 Dec. 2001.

[6] A. Putnam, S. Swanson, K. Michelson, M. Mercaldi, A. Petersen, A. Schwerin, M. Oskin, and S. J. Eggers. The microarchitecture of apipelined wavescalar processor: An rtl-based study. Technical Report TR-2005-11-02, Computer Science and Engineering, University of Washington, Nov 2005.

[7] RG. Handel-C Language Reference Manual. Celoxica Limited, http://babbage.cs.qc.edu/courses/cs345/Manuals/HandelC.pdf, 2005.

[8] K. Sankaralingam, R. Nagarajan, H. Liu, C. Kim, J. Huh, D. Burger, S. W. Keckler, and C. R. Moore. Exploiting ilp, tlp, and dlp with the polymorphous trips architecture. SIGARCH Comput. Archit. News, 31(2):422-433, 2003.

[9] J. Silc, B. Robic, and T. Ungerer. Asynchrony in parallel computing: From dataflow to multithreading. Technical report, 1997.

[10] S. Swanson. The WaveScalar Architecture. PhD thesis, University of Washington, 2006.

[11] S. Swanson, K. Michelson, A. Schwerin, and M. Oskin. Wavescalar. In Microarchitecture, 2003. MICRO-36. Proceedings. 36th Annual IEEE/ACM International Symposium on, pages 291-302, 2003.

[12] S. Swanson, A. Putnam, M. Mercaldi, K. Michelson, A. Petersen, A. Schwerin, M. Oskin, and S. Eggers. Area-performance trade-offs in tiled dataflow architectures. In Computer Architecture, 2006. 33rd International Symposium on, pages 314-326, 17-21 June 2006.

[13] N. S. Szabó and R. I. Tanaka. Residue arithmetic and its applications to computer technology. McGraw-Hill series in information processing and computers. 1967.

[14] E. Waingold, M. Taylor, D. Srikrishna, V. Sarkar, W. Lee, V. Lee, J. Kim, M. Frank, P. Finch, R. Barua, J. Babb, S. Amarasinghe, and A. Agarwal. Baring it all to software: Raw machines. Computer, 30(9):86-93, Sept. 1997. 